\title{
EXTENDING THE TORELLI MAP TO TOROIDAL COMPACTIFICATIONS OF SIEGEL SPACE
}

\author{
VALERY ALEXEEV AND ADRIAN BRUNYATE
}

\begin{abstract}
It has been known since the 1970s that the Torelli map $\mathrm{M}_{g} \rightarrow \mathrm{A}_{g}$, associating to a smooth curve its jacobian, extends to a regular map from the Deligne-Mumford compactification $\overline{\mathrm{M}}_{g}$ to the 2nd Voronoi compactification $\overline{\mathrm{A}}_{g}^{\mathrm{vor}}$. We prove that the extended Torelli map to the perfect cone (1st Voronoi) compactification $\overline{\mathrm{A}}_{g}^{\text {perf }}$ is also regular, and moreover $\overline{\mathrm{A}}_{g}^{\mathrm{vor}}$ and $\overline{\mathrm{A}}_{g}^{\text {perf }}$ share a common Zariski open neighborhood of the image of $\overline{\mathrm{M}}_{g}$. We also show that the map to the Igusa monoidal transform (central cone compactification) is not regular for $g \geq 9$; this disproves a 1973 conjecture of Namikawa.
\end{abstract}

\section{Contents}

Introduction

1. Toroidal compactifications of $\mathrm{A}_{g}$

2. Graphs and quadratic forms

3. Criteria for the regularity of the extended Torelli map

4. $\mathbb{Z}$-emms and positive cycle 2 -covers of graphs

5. Existence of $\mathbb{R}$-emms

6. Concluding remarks and generalizations

References

\section{Introduction}

The Torelli map $\mathrm{M}_{g} \rightarrow \mathrm{A}_{g}$ associates to a smooth curve $C$ its jacobian $J C$, a principally polarized abelian variety. Does it extend to a regular map $\overline{\mathrm{M}}_{g} \rightarrow \overline{\mathrm{A}}_{g}$ between the compactified moduli spaces?

For the moduli space of curves $\mathrm{M}_{g}$, a somewhat canonical choice of a compactification is provided by the Deligne-Mumford compactification $\overline{\mathrm{M}}_{g}$, which we fix for the remainder of the paper.

We note in passing that recently other compactifications $\overline{\mathrm{M}}_{g}(\alpha)$ were considered by many authors. These are $\log$ canonical models of $\overline{\mathrm{M}}_{g}$ with respect to $K_{\overline{\mathrm{M}}_{g}}+\alpha \delta$, where $\delta$ is the boundary. They also have modular interpretation. For example, for $9 / 11 \geq \alpha>7 / 10$, assuming $g \geq 3, \overline{\mathrm{M}}_{g}(\alpha)$ is the moduli spaces of curves with nodes and cusps and without elliptic tails. However, the extended map $\overline{\mathrm{M}}_{g}(\alpha) \rightarrow \overline{\mathrm{A}}_{g}$ has no chance of being regular (unless $\overline{\mathrm{M}}_{g}(\alpha)=\overline{\mathrm{M}}_{g}$ ) because curves of compact type with elliptic tails map to the interior $\mathrm{A}_{g}$, which has to be suitably modified as well.

Date: February 23, 2011; corrected: April 27 and May 25, 2011. 
For the moduli space of principally polarized abelian varieties $\mathrm{A}_{g}$, by [AMRT75] there are infinitely many choices of toroidal compactifications $\overline{\mathrm{A}}_{g}^{\tau}$, each determined by a fan $\tau$ supported on the space of positive semidefinite quadratic forms in $g$ variables, periodic w.r.t. $\operatorname{GL}(g, \mathbb{Z})$, with only finitely many orbits. There are three standard explicit choices for $\tau$, and they all have interesting geometric meanings:

(1) 1 st Voronoi fan $=$ perfect $\operatorname{cones} \tau^{\text {perf }}$,

(2) 2nd Voronoi fan $=$ Delaunay-Voronoi fan $=$ L-type domains $\tau^{\text {vor }}$,

(3) central cones $\tau^{\text {cent }}$.

The first two of these were defined by G. Voronoi in a series of papers [Vor09] on reduction theory of quadratic forms, published posthumously in 1908-9.

The 2nd Voronoi compactification appears in [Ale02] as the normalization of the main irreducible component of the moduli space $\overline{\mathrm{AP}}_{g}$ of stable semiabelic pairs $(X, \Theta)$ which provides a moduli compactification of $\mathrm{A}_{g}$. On the other hand, by [SB06] the perfect cone compactification $\overline{\mathrm{A}}_{g}^{\text {perf }}$ is the canonical model of any smooth compactification of $\mathrm{A}_{g}$, if $g \geq 12$ (and also for all $g$, if considered as stacks and relatively over Satake-Baily-Borel compactification $\overline{\mathrm{A}}_{g}^{*}$ ).

The central cones fan was introduced by Igusa [Igu67]; the corresponding toroidal compactification $\overline{\mathrm{A}}_{g}^{\mathrm{cent}}$ is the normalization of the blowup of the Satake-Baily-Borel compactification $\overline{\mathrm{A}}_{g}^{*}$ along the boundary (the "Igusa blowup").

The basic question we consider is this: for which choices of a fan $\tau$ does the Torelli map $\mathrm{M}_{g} \rightarrow \mathrm{A}_{g}$ extend to a regular map map $\overline{\mathrm{M}}_{g} \rightarrow \overline{\mathrm{A}}_{g}^{\tau}$ ? For the 2nd Voronoi fan, a positive answer was given by Mumford and Namikawa [Nam76, §18]. This prompted an extensive study of the 2nd Voronoi compactification by Namikawa [Nam76], continued in the construction of the moduli of stable semiabelic pairs $\overline{\mathrm{AP}}_{g}$ in [Ale02]. The work [Ale04] gives a modular interpretation for the extended Torelli map $\overline{\mathrm{M}}_{g} \rightarrow \overline{\mathrm{AP}}_{g}$.

Historically, the extension question for the Igusa blowup was the first one to be considered, in a pioneering 1973 paper [Nam73] of Namikawa. There, it is shown that $\overline{\mathrm{M}}_{g} \rightarrow \overline{\mathrm{A}}_{g}^{\text {cent }}$ is regular for low $g$ (the bound $g \leq 6$ is stated without proof), regular on the locus of curves with a planar dual graph, and conjectured that the map is regular for all $g$.

The question for the perfect cone compactifications was not previously considered, to our knowledge.

In this paper, we prove that the extended map is regular for the perfect cone compactification for all $g$. Much more than that, we prove that the perfect and the 2nd Voronoi compactifications share a common open neighborhood of the image of $\overline{\mathrm{M}}_{g}$. Note that in general there is a birational map $\overline{\mathrm{A}}_{g}^{\mathrm{vor}} \rightarrow \overline{\mathrm{A}}_{g}^{\text {perf }}$ which does not create new divisors. It is an isomorphism iff $g \leq 3$, and regular for $g \leq 5$. According to [ER01, ER02], this map is not regular for $g \geq 6$. Thus, for higher $g$ the two compactifications are truly different, but we prove that they are equal near the closure of the Schottky locus.

For the central cone compactification, we prove that the extended map is regular for $g \leq 6$ and is not regular for $g \geq 9$. Continuing the methods of the present paper, [ALT ${ }^{+} 10$ ] also settled the cases $g=7,8$ positively, by a lengthy computation.

The structure of the paper is as follows. In Section 1 we recall the combinatorial data for a toroidal compactification of $\mathrm{A}_{g}$, and define the fans $\tau^{\text {perf }}, \tau^{\text {cent }}, \tau^{\text {vor }}$.

In Section 2 we fix the notations for graphs and define edge-minimizing metrics, which we abbreviate to $\mathrm{emm}$, on the first cohomology group $H^{1}(G, \mathbb{Z})$ of a graph. 
In Section 3 we give a general criterion for the regularity of the extended Torelli map $\overline{\mathrm{M}}_{g} \rightarrow \overline{\mathrm{A}}_{g}^{\tau}$, and illustrate it in the case of the 2nd Voronoi compactification, as proved by Mumford and Namikawa. Then we reduce the cases of perfect cones, resp. central cones, to the existence of an $\mathbb{R}$-valued, resp. a $\mathbb{Z}$-valued emm for any graph of genus $\leq g$. We also prove that the existence of a strong $\mathbb{R}$-emm implies that $\overline{\mathrm{A}}_{g}^{\text {perf }}$ and $\overline{\mathrm{A}}_{g}^{\mathrm{vor}}$ share a common open neighborhood of the image of $\overline{\mathrm{M}}_{g}$.

In Section 4, we prove that a $\mathbb{Z}$-emm exists for any graph of genus $g \leq 6$, and does not exist for some explicit graphs of genus 9 , thus settling negatively the extension question for the central cone compactification and $g \geq 9$.

In Section 5, we prove that a strong $\mathbb{R}$-emm exists for any graph, thus proving the regularity of $\overline{\mathrm{M}}_{g} \rightarrow \overline{\mathrm{A}}_{g}^{\text {perf }}$ and the statement about a common neighborhood.

In the concluding Section 6 we discuss some possible extensions of out results.

Acknowledgments. The first author was partially supported by NSF under DMS-0901309. The subject of the paper was the topic of a VIGRE research group at the University of Georgia in the Fall of 2010, led by the first author. We would like to thank the participants of the group for many discussions, and to acknowledge NSF's VIGRE support under DMS0738586. We also thank the referee for very helpful suggestions for improvements.

\section{Toroidal compactifications of $\mathbf{A}_{g}$}

Here, $\mathrm{A}_{g}$ stands for the moduli space of principally polarized abelian varieties. The theory of its toroidal compactifications over $\mathbb{C}$ was developed by Mumford and his coworkers in [AMRT75]; [FC90] contains an extension to the arithmetic case, over $\mathbb{Z}$. It is parallel to the theory of ordinary toric varieties.

As in toric geometry, there are two dual lattices, $M$ (for monomials) and $N$ (for 1parameter subgroups in the torus). The real vector space $N_{\mathbb{R}}$ is the ambient space for a fan $\tau$, and $M_{\mathbb{R}}$ is the ambient space for polyhedra. For compactifications of $\mathrm{A}_{g}$, one fixes a free abelian group $\Lambda \simeq \mathbb{Z}^{g}$. Then $M=\operatorname{Sym}^{2} \Lambda$, and $N=\Gamma^{2} \Lambda^{*}$ is the dual abelian group, the second divided power of $\Lambda^{*}$.

Let us choose a basis $f_{1}, \ldots, f_{g}$ of $\Lambda$ and a dual basis $f_{1}^{*}, \ldots, f_{g}^{*}$ of $\Lambda^{*}$, so that $\left(f_{i}^{*}, f_{j}\right)=$ $\sigma_{i j}$. Then the elements of the lattice $M=\operatorname{Sym}^{2} \Lambda$ are integral homogeneous quadratic functions $q=\sum_{i<j} q_{i j} f_{i} f_{j}, q_{i j} \in \mathbb{Z}$, on $\Lambda^{*}$. These correspond to symmetric half-integral $g \times g$ matrices $A=\left(a_{i j}\right)$, which means that $a_{i i} \in \mathbb{Z}$ and $a_{i j} \in \frac{1}{2} \mathbb{Z}$ for $i \neq j$. Equivalently, $2 A$ is the matrix of an even integral bilinear form.

The elements of $N=\Gamma^{2} \Lambda^{*}$ are integral tensors $\sum b_{i j} f_{i}^{*} \otimes f_{j}^{*}$ symmetric under the involution $f_{i}^{*} \otimes f_{j}^{*} \mapsto f_{j}^{*} \otimes f_{i}^{*}$. Thus, $N$ can be identified with the space of symmetric integral matrices $B=\left(b_{i j}\right), b_{i j} \in \mathbb{Z}$.

Both $M$ and $N$ can be considered as the lattices in the space of real symmetric $g \times g$ matrices. They are dual with respect to the inner product $(A, B)=\operatorname{Tr} A B$.

Now let $C$ be the open cone $C$ in $N_{\mathbb{R}}$ consisting of positive-definite symmetric real matrices. This cone is self-dual with respect to the above inner product. One fixes its "closure" $\bar{C}$. To be precise, $\bar{C}$ is the real cone spanned by semi definite positive symmetric matrices $B \geq 0$ with rational radical (i.e., the null space of $B$ has to have a basis of vectors with rational coordinates). 
Then a toroidal compactification $\overline{\mathrm{A}}_{g}^{\tau}$ of $\mathrm{A}_{g}$ is defined by a fan $\tau$ (i.e. a collection of finitely generated rational cones, closed under taking faces) in $N_{\mathbb{R}}$ satisfying the following properties:

(1) $\operatorname{Supp} \tau=\bar{C}$.

(2) The natural $\mathrm{GL}(g, \mathbb{Z})$-action on $N_{\mathbb{R}}$ sends cones of $\tau$ to cones of $\tau$.

(3) There are only finitely many orbits of cones under this action.

The following are three standard fans corresponding to three standard toroidal compactifications of $\mathrm{A}_{g}$ :

The perfect cones fan $\tau^{\text {perf }}$, otherwise known as the 1 st Voronoi fan. The cones are defined to be the cones over the faces of the convex hull of $N \cap(\bar{C} \backslash 0)$. By a result of Barnes and Cohn [BC76], the vertices of Conv $N \cap(\bar{C} \backslash 0)$ (that is, the rays of $\tau^{\text {perf }}$ ) are of the form $a^{* 2}$, where $a^{*}=\sum a_{i} f_{i}^{*}$ is an integral primitive (i.e. indivisible) nonzero element of $\Lambda^{*}$. Thus, every perfect cone $\sigma$ has the form $\sigma=\sum_{s} \mathbb{R}_{\geq 0} a_{s}^{* 2}$ for some collection $\left\{a_{s}^{*}\right\} \subset \Lambda^{*} \backslash 0$.

For $q \in \operatorname{Sym}^{2} \Lambda_{\mathbb{R}}$, one has $\left(q, a^{* 2}\right)=q\left(a^{*}\right)$, the value of the quadratic function $q$ at the integral point $a^{*} \in \Lambda^{*}$. Thus, if $\sigma^{\vee}$ is the dual cone in $M_{\mathbb{R}}$, then the elements of the interior $\left(\sigma^{\vee}\right)^{0}$ are the positive definite quadratic functions which attain the minimum on the same finite subset $\left\{a_{s}^{*}\right\}$.

In particular, for a maximal cone $\sigma \in \tau^{\text {perf }}$, the cone $\sigma^{\vee}$ is generated by one quadratic function which is determined up to a multiple by the set of its minimal integral nonzero vectors. Such quadratic forms are called perfect, hence the name of this fan.

The second Voronoi fan $\tau^{\text {vor }}$, sometimes referred to as Delaunay-Voronoi fan, or $L$ type decomposition. The locally closed cones $\tau^{0}$ of this fan consist of quadratic forms which define the same Delaunay decomposition of $\Lambda_{\mathbb{R}} / \Lambda$.

The central cones fan $\tau^{\text {cent }}$, corresponding to the normalization of the Igusa blowup. Let $Q$ be the convex hull of $C \cap(M \backslash 0)$. This is an infinite polyhedron whose faces are (finite) polytopes. The fan $\tau^{\text {cent }}$ is the dual fan of $Q$. The vertices of Conv $(C \cap(M \backslash 0))$ are called central quadratic forms. Note that they are integral by definition. The corresponding cones of $\tau^{\text {cent }}$ are maximal-dimensional central cones.

Each of the fans $\tau^{\text {perf }}, \tau^{\text {vor }}, \tau^{\text {cent }}$ admits a strictly convex support function, (1) and (3) by definition and (2) by [Ale02]. Hence, the compactifications $\overline{\mathrm{A}}_{g}^{\mathrm{perf}}, \overline{\mathrm{A}}_{g}^{\mathrm{vor}}, \overline{\mathrm{A}}_{g}^{\mathrm{cent}}$ are projective by Tai's criterion [AMRT75, IV.2].

\section{Graphs and quadratic forms}

$G$ will denote a graph with edges $e_{i}, i=1, \ldots, m$ and vertices $v_{j}, j=1, \ldots, n$. We allow multiple edges and loops. We fix an orientation of edges. Then we have the usual boundary homomorphism

$$
\partial: C_{1}(G, \mathbb{Z})=\oplus_{i} \mathbb{Z} e_{i} \rightarrow C_{0}(G, \mathbb{Z})=\oplus_{j} \mathbb{Z} v_{j}, \quad \partial e_{i}=\operatorname{end}\left(e_{i}\right)-\operatorname{beg}\left(e_{i}\right)
$$

The kernel of this map is the space of cycles $H_{1}(G, \mathbb{Z})$ and the cokernel is $H_{0}(G, \mathbb{Z})$. We will assume $G$ to be connected, so that $H_{0}(G, \mathbb{Z})=\mathbb{Z}$. Dually, we have the homomorphism

$$
d: C^{0}(G, \mathbb{Z})=\oplus_{j} \mathbb{Z} v_{j}^{*} \rightarrow C^{1}(G, \mathbb{Z})=\oplus_{i} \mathbb{Z} e_{i}^{*}, \quad d v_{j}^{*}=\sum_{v_{j}=\operatorname{end}\left(e_{i}\right)} e_{i}^{*}-\sum_{v_{j}=\operatorname{beg}\left(e_{i}\right)} e_{i}^{*}
$$

with kernel $H^{0}(G, \mathbb{Z})=\mathbb{Z}$ and cokernel $H^{1}(G, \mathbb{Z})$. 
Definition 2.1. We call the elements $e_{i}^{*}$ in $H^{1}(G, \mathbb{Z})$ coedges, to distinguish them from the edges $e_{i} \in C_{1}(G, \mathbb{Z})$. Thus, coedges are cocycles and edges are chains.

Since any graph is homotopy equivalent to a graph with one vertex and $g$ loops for some $g \geq 0$, called the genus of $G, H_{1}(G, \mathbb{Z})$ and $H^{1}(G, \mathbb{Z})$ are free abelian groups of rank $g$, dual to each other.

Lemma 2.2. One has the following:

(1) The elements $e_{i}^{*}$ span $H^{1}(G, \mathbb{Z})$.

(2) $e_{i}^{*}=0$ iff the edge $e_{i}$ is a bridge in $G$.

(3) The graph $G$ is a simple loop (a graph with one vertex and one edge) or is loopless and is 2-connected $\Longleftrightarrow$ the edges can not be divided into two disjoint groups $I_{1} \sqcup I_{2}$ such that

$$
H^{1}(G, \mathbb{Z})=\left\langle e_{i_{1}}^{*}\right\rangle \oplus\left\langle e_{i_{2}}^{*}\right\rangle, \quad i_{s} \in I_{s} .
$$

Proof. (1) and (2) are obvious. For (3), consider a partition of edges $I_{1} \sqcup I_{2}$, and denote by $G_{s}, s=1,2$, the graph formed by the edges of $I_{s}$. Note that the zero set of $I_{s}$ in $H_{1}(G)$ is $H_{1}\left(G_{3-s}\right)$. Since $e_{i}^{*}$ span $H^{1}(G, \mathbb{Z})$, the condition of $(3)$ is that the intersection is zero, equivalently that $H_{1}(G)$ is spanned by $H_{1}\left(G_{1}\right)$ and $H_{1}\left(G_{2}\right)$, i.e. every simple cycle in $G$ lies entirely either in $G_{1}$ or in $G_{2}$. If $G$ has a loop (but $G$ is not a loop itself) or $G$ is not 2-connected, then obviously there is such a decomposition. Vice versa, given such a decomposition, every vertex in $G_{1} \cap G_{2}$ is a cut of $G$ or is a vertex of a loop, so $G$ is not 2 -connected or it has a loop.

The following lemma gives explicit $\mathbb{Z}$-bases for $H_{1}(G, \mathbb{Z})$ and $H^{1}(G, \mathbb{Z})$.

Lemma 2.3. For a collection of edges $e_{i}, i \in I \subset\{1, \ldots, m\}$, the following conditions are equivalent:

(1) $e_{i}^{*}$ form an $\mathbb{R}$-basis of $H^{1}(G, \mathbb{R})$.

(2) $e_{i}^{*}$ form a $\mathbb{Z}$-basis of $H^{1}(G, \mathbb{Z})$.

(3) The complement of $\left\{e_{i}\right\}$ is a spanning tree $T$ of $G$.

If either of these conditions is satisfied then there exists a basis of $H_{1}(G, \mathbb{Z})$ of the form

$$
f_{i}=e_{i}+\sum_{e_{s} \in T} b_{i s} e_{s}, \quad b_{i s}=0, \pm 1, i \in I .
$$

Proof. Of course, (2) implies (1). Let us prove (1) $\Rightarrow(3)$. Note that $|I|=g$.

By the Euler's formula, $g(G)=m+1-n$ and $\chi(G)=1-g$. Since the graph $G^{\prime}=$ $G \backslash\left\{e_{i}, i \in I\right\}$ has the same vertices and $g$ fewer edges, we have $\chi\left(G^{\prime}\right)=1$. Then either $G^{\prime}$ is connected and is a tree, or else $G^{\prime}$ is disconnected and has a nonzero loop, call it $\ell$. Then for all $i \in I$ we have $e_{i}^{*}(\ell)=0$, hence $\left\{e_{i}^{*}, i \in I\right\}$ is not a basis of $H^{1}(G, \mathbb{R})$. QED.

$(3) \Rightarrow(2)$. We prove this by constructing a dual basis $\left\{f_{i}\right\}$ in $H_{1}(G, \mathbb{Z})$ to the set $\left\{e_{i}^{*}\right\}$. Since $T$ is a tree, for each $j$ there exists a unique path in $T$ from the end to the beginning of $e_{i}$. In other words, there exists a unique $f_{i} \in H_{1}(G, \mathbb{Z})$ which can be written as

$$
f_{i}=e_{i}+\sum_{e_{s} \in T} b_{i s} e_{s}, \quad b_{i s}=0, \pm 1 .
$$

Then it is clear that $e_{i}^{*}\left(f_{k}\right)=1$ if $j=k$ and 0 otherwise. Thus, $\left\{e_{i}^{*}\right\}$ and $\left\{f_{i}\right\}$ are dual bases in $H^{1}(G, \mathbb{Z})$ and $H_{1}(G, \mathbb{Z})$.

Definition 2.4. An edge-minimizing metric, abbreviated to emm, of a graph $G$ is a quadratic form $q \in \operatorname{Sym}^{2} H_{1}(G)$ such that 
(1) $q>0$, i.e. $q$ is positive definite.

(2) $q\left(e_{i}^{*}\right)=1$ for each edge $e_{i}$ which is not a bridge (i.e. for each $e_{i}^{*} \neq 0$ ).

(3) $q\left(v^{*}\right) \geq 1$ for any $v^{*} \in H^{1}(G, \mathbb{Z}) \backslash 0$.

A strong edge-minimizing metric, in addition, satisfies the following: if $q\left(v^{*}\right)=1$ for some $v^{*} \in H^{1}(G, \mathbb{Z})$ then $\pm v^{*}$ is a coedge.

In other words, $q$ is a metric on the lattice $H^{1}(G, \mathbb{Z})$ and the nonzero $\pm e_{i}^{*}$ are among the shortest (resp. exactly the shortest) integral vectors in this metric.

We will distinguish between $q \in \operatorname{Sym}^{2} H_{1}(G, R)$, where $R$ is $\mathbb{Z}, \mathbb{Q}$, or $\mathbb{R}$. We will call these $\mathbb{Z}$-emm, $\mathbb{Q}$-emm, $\mathbb{R}$-emm respectively. There will be no difference between $\mathbb{Q}$-emms and $\mathbb{R}$-emms for our purposes.

Definition 2.5. By Lemma 2.2(3), one has $H^{1}(G, \mathbb{Z})=\oplus_{k} H^{1}\left(G_{k}, \mathbb{Z}\right)$ for some graphs $G_{k}$ so that each $G_{k}$ is either a simple loop or loopless and 2-connected, and so that each nonzero $e_{i}^{*}$ lies in one of the direct summands. We may call $G_{k}$ irreducible components of $G$.

Lemma 2.6. There exists a $(\mathbb{Z}, \mathbb{Q}$, or $\mathbb{R})$ emm for a graph $G \Longleftrightarrow$ there exist emms for each irreducible component $G_{k}$.

Proof. The restriction $q_{k}$ of an emm $q$ to each $H^{1}\left(G_{k}, \mathbb{Z}\right)$ is an emm. Vice versa, given emms $q_{k}$ for graphs $G_{k}$, we can take $q=\sum q_{k}$ to be an emm for $G$.

Lemma 2.7. To construct a $(\mathbb{Z}, \mathbb{Q}$, or $\mathbb{R})$ emm for a graph $G$, it is sufficient to construct an emm for several related cubic bridgeless graphs.

A remark concerning our terminology: cubic is the same as trivalent, and bridgeless is the same as 2-connected.

Proof. By the above Lemma, it is sufficient to construct an emm for each irreducible component $G_{k}$. If $G_{k}$ is a loop then $q=x^{2}$ is a $\mathbb{Z}$-emm. So assume $G_{k}$ is not a loop.

Removing vertices of degree 2 and replacing the adjacent two edges by a single edge results in reducing some duplication in the set $\left\{e_{i}^{*}\right\}$. Next, we inductively insert an edge into a vertex of degree $\geq 4$, until we get to a cubic graph $G_{k}^{\prime}$. Doing so does not change $H_{1}$ but adds more vectors $e_{i}^{*}$, so the condition for $G_{k}^{\prime}$ is stronger than for $G_{k}$.

\section{Criteria for the regularity of the extended Torelli map}

As in Section 1, we fix a lattice $\Lambda \simeq \mathbb{Z}^{g}$, a fan $\tau$, and a corresponding toroidal compactification $\overline{\mathrm{A}}_{g}^{\tau}$. We also consider a graph $G$ of genus $a \leq g$ and write its homology as a quotient $\Lambda \rightarrow H_{1}(G, \mathbb{Z})$. This gives a cotorsion embedding of $N(G):=\Gamma^{2} H^{1}(\Gamma, \mathbb{Z})$ into $N=\Gamma^{2} \Lambda^{*}$. We work either over a field or over $\mathbb{Z}$.

Definition 3.1. We will denote by $S(G)$ the set of nonzero vectors $e_{i}^{* 2}$ in $N(G)$.

Theorem 3.2 (General criterion). The Torelli map $\overline{\mathrm{M}}_{g} \rightarrow \overline{\mathrm{A}}_{g}^{\tau}$ is regular in a neighborhood of a stable curve $[C]$ iff for the dual graph $G(C)$ there exists a cone $\sigma$ in the fan $\tau$ such that $S(G) \subset \sigma$.

Proof. The stacks $\left(\overline{\mathrm{M}}_{g}, \partial \overline{\mathrm{M}}_{g}\right)$ and $\left(\overline{\mathrm{A}}_{g}^{\tau}, \partial \overline{\mathrm{A}}_{g}^{\tau}\right)$ are toroidal. The second one by definition, and the first one because it is a smooth stack of dimension $3 g-3$ and the boundary divisors have normal crossings. 
Thus, in a neighborhood of a boundary point $[C]$ of $\overline{\mathrm{M}}_{g}$ corresponding to a stable curve, $\overline{\mathrm{M}}_{g}$ is a toroidal stack modeled on $\left(\mathbb{A}^{1}, 0\right)^{m} \times \mathbb{G}_{m}^{3 g-3-m}$, where $m$ is the the number of edges of the dual graph $\Gamma$ of $C$, and $\mathbb{G}_{m}$ is the multiplicative group. This corresponds to a standard $m$-dimensional cone in $\mathbb{R}^{3 g-3}$ generated by the first $m$ coordinate vectors which are in a bijection with the edges $e_{i}$ of $\Gamma$.

By the Picard-Lefshetz monodromy formula, near the boundary the Torelli map is described by the linear map sending the vector $e_{i}$ to $\left(e_{i}^{*}\right)^{2} \in N$. We conclude the proof by applying a well-known criterion of regularity for toroidal varieties saying that the rational map is regular iff every cone of the first fan maps into a cone in the second fan.

The coarse moduli spaces are locally finite Galois quotients of appropriate toroidal neighborhoods for $\overline{\mathrm{M}}_{g}, \overline{\mathrm{A}}_{g}^{\mathrm{vor}}$. The regularity of the rational map is unaffected by such Galois covers. Hence, the result for the coarse moduli spaces is the same as for the stacks.

Lemma 3.3. The map $\overline{\mathrm{M}}_{g} \rightarrow-\overline{\mathrm{A}}_{g}^{\tau}$ is regular on an open union of strata of $\overline{\mathrm{M}}_{g}$.

Proof. For two stable curves $C, C^{\prime}$, the stratum of $C$ is in the closure of the stratum of $C^{\prime}$ iff $G$ is a contraction of $G^{\prime}$. Then $H_{1}\left(G^{\prime}, \mathbb{Z}\right) \rightarrow H_{1}(G, \mathbb{Z})$, the lattice $N(G)$ is a cotorsion sublattice in $N\left(G^{\prime}\right)$, and $S(G) \subset S\left(G^{\prime}\right)$. Thus, if $S\left(G^{\prime}\right) \subset \sigma$ then $S(G) \subset \sigma . S(G)$ also lies in a cone of the induced fan on $N(G)_{\mathbb{R}}$.

As a first application, we reprove the following result of Mumford and Namikawa, cf. $[$ Nam76, §18].

Theorem 3.4. The map $\overline{\mathrm{M}}_{g} \rightarrow \overline{\mathrm{A}}_{g}^{\mathrm{vor}}$ is regular.

Proof. This immediately follows from Theorem 3.2, Lemma 2.3, and the following well known elementary fact about the "dicing" 2nd Voronoi cones, cf. [ER94].

Lemma 3.5 (Dicings). Let $v_{i}^{*} \in \Lambda^{*}, i \in I$, be finitely many nonzero primitive vectors. Then the following conditions are equivalent:

(1) $\left\{v_{i}^{* 2}\right\}$ lie in the same 2nd Voronoi cone,

(2) $\sum \mathbb{R}_{\geq 0} v_{i}^{* 2}$ is a 2nd Voronoi cone,

(3) Any linearly independent subset $\left\{v_{j}^{*}\right\}, j \in J \subset I$, is a $\mathbb{Z}$-basis of $\Lambda^{*} \cap \sum \mathbb{R} v_{j}^{*}$.

Remark 3.6. The systems of vectors in the above lemma are known by various names: totally unimodular systems of vectors, dicings, regular matroids. The "dicing" refers to the corresponding Delaunay decomposition of $\Lambda_{\mathbb{R}}$ periodic w.r.t. $\Lambda$. It is given by "dicing" the vector space $\Lambda_{\mathbb{R}}$ by the parallel systems of hyperplanes $\left\{v_{i}^{*}=n_{i} \in \mathbb{Z}\right\}$.

Seymour's classification theorem on regular matroids, which can be found in [Oxl92], says that all regular matroids are graphic, cographic, a special matroid $R_{10}$, or can be obtained from these by a sort of "tensor product".

The regular matroids above, corresponding to $\left\{e_{i}^{*} \in H^{1}(G, \mathbb{Z})\right\}$, are the cographic matroids. This gives the combinatorial description of the toroidal Torelli map. By [ER94, 4.1] every dicing 2nd Voronoi cone is simplicial. Thus, the open neighborhood of $\operatorname{im} \bar{M}_{g}$ in the stack $\overline{\mathrm{A}}_{g}^{\mathrm{vor}}$ corresponding to the cographic dicing cones has at worst abelian quotient singularities.

We now turn to the cases of perfect and central cones.

Theorem 3.7. (1) The Torelli map $\mathrm{M}_{g} \rightarrow \overline{\mathrm{A}}_{g}^{\text {perf }}$ is regular in a neighborhood of a stable curve $[C]$ iff the dual graph $G(C)$ has an $\mathbb{R}$-emm. 
(2) Moreover, if every graph $G$ of genus $\leq g$ has a strong $\mathbb{R}$-emm then $\overline{\mathrm{A}}_{g}^{\text {perf }}$ and $\overline{\mathrm{A}}_{g}^{\text {vor }}$ share a common open neighborhood of the image of $\overline{\mathrm{M}}_{g}$.

Proof. (1) By the description of the perfect fan given in Section $1, e_{i}^{* 2}$ lie in a perfect cone iff they are edges of some perfect cone $\sigma$. This means that there exists a positive definite quadratic form $q$ such that the nonzero $e_{i}^{*}$ are among the shortest integral vectors w.r.t. $q$. This is our definition of an $\mathbb{R}$-emm.

(2) By the above, the strata of $\overline{\mathrm{A}}_{g}^{\mathrm{vor}}$ corresponding to the cographic regular matroids gives a Zariski open neighborhood $U$ of the image of $\overline{\mathrm{M}}_{g}$. We want to show that each of these 2nd Voronoi cones is also a perfect cone. This means that there exists a $q>0$ such that the nonzero $\pm e_{i}^{*}$ are exactly the shortest integral vectors w.r.t. $q$. This is our definition of a strong $\mathbb{R}$-emm.

Theorem 3.8. The Torelli map $\mathrm{M}_{g} \rightarrow \overline{\mathrm{A}}_{g}^{\mathrm{cent}}$ is regular in a neighborhood of a stable curve $[C]$ iff the dual graph $G(C)$ has a $\mathbb{Z}$-emm.

Proof. Applying Theorem 3.2, if the map is regular then $\left\{e_{i}^{* 2}\right\}$ lie in the same central cone $\sigma$, which we can pick to be maximal-dimensional. The corresponding dual cone $\sigma^{\vee}$ is 1dimensional and is spanned by a central form $q \in M$. This is an integral positive definite form characterized by the following property: for any $f \in \sigma$ and any other integral positive definite form $q^{\prime} \in M$ one has $(q, f) \leq\left(q^{\prime}, f\right)$. Since every $e_{i}^{*} \neq 0$ is a primitive vector in $\Lambda^{*}$, there exist a $q^{\prime}$ with $\left(q^{\prime}, e_{i}^{* 2}\right)=q^{\prime}\left(e_{i}^{*}\right)=1$. Therefore, $q\left(e_{i}^{*}\right)=1$ for all nonbridge edges $e_{i}$, and so $q$ is a $\mathbb{Z}$-emm.

Vice versa, if $q$ is a $\mathbb{Z}$-emm of $G$ then $1=q\left(e_{i}^{*}\right) \leq q^{\prime}\left(e_{i}^{*}\right)$ for any $q^{\prime} \in M \cap C \backslash 0$, so $\left\{e_{i}^{* 2}\right\} \subset \sigma$ for any cone $\sigma^{\vee}$ containing $q$.

Lemma 3.9. The subset of $\overline{\mathrm{M}}_{g}$ of curves admitting a $\mathbb{Z}$-emm, resp. $\mathbb{R}$-emm, is an open union of strata (cf. Lemma 2.7).

Proof. Using the notations of the proof of Lemma 3.3, the restriction of an emm on $H^{1}\left(G^{\prime}, \mathbb{Z}\right)$ to $H^{1}(G, \mathbb{Z})$ is an emm for $G$.

\section{4. $\mathbb{Z}$-emms and positive cycle 2 -covers of graphs}

Lemma 4.1. Let $q$ be a $\mathbb{Z}$-emm of a graph $G$. Then the lattice $\left(H^{1}(G, \mathbb{Z}), 2 q\right)$ is a direct sum of the standard root lattices $A_{n}, D_{n}(n \geq 4), E_{n}(n=6,7,8)$.

If, in addition, $G$ has no loops and is 2-connected, then the root lattice $\left(H^{1}(G, \mathbb{Z}), 2 q\right)$ is irreducible, i.e. it is a single copy of $A_{n}, D_{n}$, or $E_{n}$.

Proof. The bilinear form $2 q$ is integral, symmetric, positive definite, and even. The set $R$ of vectors $r$ with $r^{2}=2$ spans $H^{1}(G, \mathbb{Z})$ since it contains $e_{i}^{*}$. Thus, $R$ is a simply laced root system, which must be a direct sum of $A_{n}, D_{n}, E_{n}$ by a standard classification.

If $\left(H^{1}(G, \mathbb{Z}), 2 q\right)$ is not irreducible then it splits as a direct $\operatorname{sum}\left\langle e_{i_{1}}^{*}\right\rangle \oplus\left\langle e_{i_{2}}^{*}\right\rangle$ for some partition $I_{1} \sqcup I_{2}$ of edges. Then $G$ is not 2-connected loopless by Lemma 2.2.

Recall that a graph is called projective planar if it admits an an embedding into $\mathbb{R P}^{2}$. The main result of this section is the following.

Theorem 4.2. Let $G$ be a 2-connected loopless graph. Then

(1) $G$ has a $\mathbb{Z}$-emm of type $A_{g} \Longleftrightarrow G$ is planar. 
(2) For $g \geq 4, G$ has a $\mathbb{Z}$-emm of type $D_{g} \Longleftrightarrow G$ is projective planar. Moreover, the directions $\Leftarrow$ hold for any graph.

Remark 4.3. The direction $\Leftarrow$ of $(1)$ is due to Namikawa [Nam73, Prop.5].

Corollary 4.4. Every graph of genus $g \leq 6$ admits a $\mathbb{Z}$-emm.

Proof. The well-known Kuratowski theorem says that a graph is not planar iff it contains a subgraph homeomorphic to $K_{5}$ or $K_{3,3}$. There is a similar theorem of Archdeacon [Arc80, Arc81] for projective planar graphs which has a much longer list of 103 minimal counterexamples. All of those graphs have genus $\geq 7$, except for a single graph $G_{1}$. The existence of a $\mathbb{Z}$-emm for $G_{1}$ can be easily established by a direct, although quite lengthy, computation.

We note that [Arc80, Arc81] is concerned with graphs without loops and and multiple edges. But a loop just adds a single $\mathbb{Z}$ summand to $H_{1}(G, \mathbb{Z})$, and the multiple edges do not affect (projective) planarity.

Remark 4.5. By extending this method, $\left[\mathrm{ALT}^{+} 10\right]$ proves the existence of a $\mathbb{Z}$-emm for every graph of genus 7 and 8 . This amounts to checking all the cubic genus 6 and 7 graphs from Archdeacon's list and the cubic graphs obtained from them by adding one or two edges.

Corollary 4.6. For any $g \geq 9$, there exists a graph $G$ of genus $g$ which does not admit a $\mathbb{Z}$-emm.

Proof. Since $\mathbb{Z}$-emms of type $E_{n}$ only appear for $g=6,7,8$, in genus $g \geq 9$ it is sufficient to take any 2-connected loopless graph containing a graph from Archdeacon's list [Arc80, Arc81] as a subgraph. For example, the graph of genus 9 in Figure 1 contains the minimal nonplanar graph of genus 6 from [Arc80, Arc81]: For $g>9$, one can obtain $G$ from it by

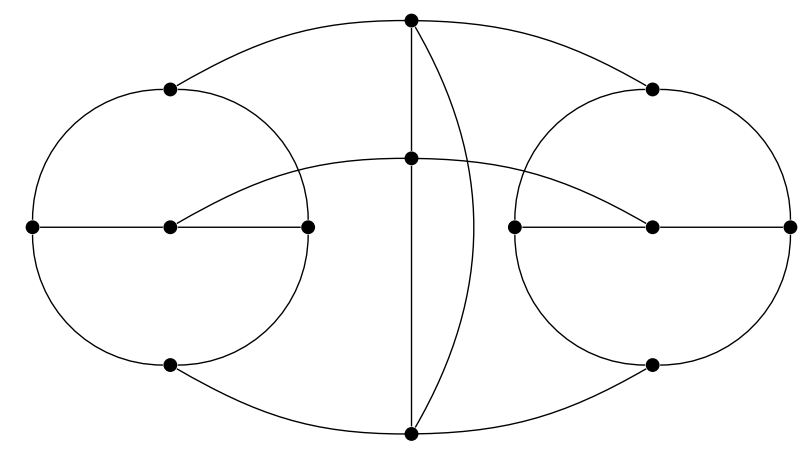

Figure 1. A graph of genus 9 without a $\mathbb{Z}$-emm

adding $g-9$ edges.

Joe Tennini pointed out to us that the list in [Arc80, Arc81] contains a graph with 7 vertices. This implies that the complete graph $K_{n}$ is not projective planar for $n \geq 7$ and does not admit a $\mathbb{Z}$-emm since $g\left(K_{7}\right)=15$.

The basic idea of our proof of Theorem 4.2 is the following. We can assume that $G$ is bridgeless, by contracting the bridges. A cycle 2-cover of $G$ is a collection of cycles $c_{k}$ 
such that every edge appears in $\cup c_{k}$ exactly twice. (A long standing conjecture of SzekeresSeymour says that every bridgeless graph has such a cycle 2-cover. We don't need the validity of this conjecture for our proof).

Now let us say $\left\{c_{k}\right\}$ is a cycle 2-cover, and consider the quadratic form $q=\frac{1}{2} \sum c_{k}^{2}$. Then $q$ is integral and since every $e_{i}^{2}$ appears in $q$ with coefficient 1 , one has $q\left(e_{i}^{*}\right)=\left(q, e_{i}^{* 2}\right)=1$. However, in general $q$ is only positive semi definite.

Definition 4.7. A cycle 2-cover $\left\{c_{k}, k=1, \cdots N\right\}$ of a graph is called positive if the quadratic form $q=\frac{1}{2} \sum_{k=1}^{N} c_{k}^{2}$ is positive definite.

Cycle 2-covers are closely related to embeddings of graphs into closed topological surfaces. Given an embedding $G \hookrightarrow S^{2}$, resp. $G \hookrightarrow \mathbb{R}^{2}$, we will construct a $\mathbb{Z}$-emm of type $A_{g}$, resp. $D_{g}$, on $H^{1}(G, \mathbb{Z})$. Then we will prove the converse by using the fact that the quadratic forms $A_{n}$ and $D_{n}$ can be written as sums of $\geq n$ squares of integral linear forms.

So let $\left\{c_{k}\right\}$ be a cycle 2 -cover. Divide each $c_{k}$ into a sum of simple (not repeating vertices) cycles $d_{\ell}$. For each $d_{\ell}$, take a copy $D_{\ell}$ of a 2 -disk and identify its boundary with $d_{\ell}$. Glue these disks along the edges $e_{i}$. The result is a closed surface $X$ which may have isolated singular points at some vertices, as follows.

For a vertex $v$, consider a simple cycle $d_{\ell}$ in the given 2-cover that goes through $v$. We constructed $X$ by gluing the boundary of a disk $D_{\ell}$ to $d_{\ell}$. In $X$ there is a neighboring disk that also contains $v$; continue from disk to disk until you have made a full circle around $v$. If these are all the $d_{\ell}$ containing $v$ then $X$ is smooth at $v$. In general, there will be several such full circles, and so $X$ is obtained from a smooth closed surface $\widetilde{X}$ by gluing together several points to such bad vertices $v$.

Theorem 4.8. Let $G$ be a loopless 2-connected graph admitting a cycle double cover $\left\{c_{k}\right\}$. Then $\left\{c_{k}\right\}$ is positive $\Longleftrightarrow X=S^{2}$ or $\mathbb{R P}^{2}$.

Proof. Assume that $q>0$. First, we claim that $X$ has no singular points, i.e. that $X=\widetilde{X}$. Assuming the opposite, let $X^{\prime}$ be the surface obtained from $X$ by normalizing at a single singular point $v$, and let $G^{\prime}$ be the preimage of $G$ in $X^{\prime}$. Since $G$ is 2-connected, $G^{\prime}$ is a connected graph. Since $G^{\prime}$ has the same edges as $G$ but more vertices, we have $g\left(G^{\prime}\right)<g(G)$, and $H_{1}\left(G^{\prime}\right) \subset H_{1}(G)$ is a proper subspace. But $q$ is the same sum of squares of elements of $H_{1}\left(G^{\prime}\right)$, so it can not be positive definite.

Next, the Euler characteristic of the smooth surface $X$ is

$$
\chi(X)=N-E+V=N-(E-V+1)+1=N-g+1
$$

Since $q$ is positive definite, $N \geq g$. Hence, $\chi(X) \geq 1$. There are only two smooth closed surfaces with $\chi \geq 1$ : $S^{2}(\chi=2, N=g+1)$ and $\mathbb{R P}^{2}(\chi=1, N=g)$.

Now, for the opposite direction. If $X=S^{2}$ then $G$ divides the sphere into $g+1$ regions with boundaries $c_{k}$. These obviously generate $H_{1}(G, \mathbb{Z})$, so $q>0$.

In the case $X=\mathbb{R} \mathbb{P}^{2}$, let $\pi: S^{2} \rightarrow \mathbb{R P}^{2}$ be the $2: 1$ cover, and let $G^{\prime}=\pi^{-1}(G)$. Because $\pi_{*} H_{1}\left(G^{\prime}, \mathbb{R}\right)=H_{1}(G, \mathbb{R})$ and the cycles on $S^{2}$ generate $H_{1}\left(G^{\prime}, \mathbb{R}\right)$, the cycles $c_{k}$ generate $H_{1}(G, \mathbb{R})$. Hence, $\sum c_{k}^{2}$ is positive definite.

Proof of Theorem 4.2. (1) Let $G$ be an arbitrary graph with an embedding $G \subset S^{2}$. If $G$ is not connected, we add bridges to make it connected. Then the set $S^{2} \backslash G$ is a union of $g+1$ regions bounded by the cycles $c_{k}$ which can be given compatible orientations. Then $H_{1}(G, \mathbb{Z})=\mathbb{Z}^{g+1} / \mathbb{Z} \sum c_{k}$, and the dual lattice $H^{1}(G, \mathbb{Z})$ is the hyperplane $\left\{\left(n_{k}\right) \in \mathbb{Z}^{g+1} \mid\right.$ $\left.\sum n_{k}=0\right\}$. The quadratic form $2 q=\sum c_{k}^{2}$ is the restriction of the standard Euclidean form on $\mathbb{Z}^{g+1}$ to $H^{1}(G, \mathbb{Z})$. This is the standard definition of the $A_{g}$ lattice. Each nonbridge edge 
$e_{i}$ belongs to precisely two 2 -cells $k_{1}, k_{2}$. Then $\pm e_{i}^{*}=c_{k_{1}}^{*}-c_{k_{2}}^{*}$, where $\left\{c_{k}^{*}\right\}$ is the Euclidean basis of $\mathbb{Z}^{g+1}$.

Vice versa, suppose that a 2 -connected loopless graph $G$ has a $\mathbb{Z}$-emm of type $A_{g}$. Note that the quadratic form of $A_{g}$ is a sum of $g+1$ squares of integral linear functions:

$$
2 q=2 \sum_{i=1}^{g} x_{i}^{2}-2 \sum_{i=1}^{g-1} x_{i} x_{i+1}=x_{1}^{2}+\left(x_{1}-x_{2}\right)^{2}+\ldots\left(x_{g-1}-x_{g}\right)^{2}+x_{g}^{2}
$$

Consider the $g+1$ cycles $c_{k}$ in $H_{1}(G, \mathbb{Z})$ corresponding to these linear terms. We claim that for each edge $e_{i}$ of $G$ we have $c_{k}\left(e_{i}^{*}\right)=0$ or \pm 1 . Indeed, if $\left|c_{k}\left(e_{i}^{*}\right)\right| \geq 2$ then for $q=\frac{1}{2} \sum_{k} c_{k}^{2}$ we have $q\left(e_{i}^{*}\right) \geq 2$, which contradicts our assumption $q\left(e_{i}^{*}\right)=1$. Thus, for each edge there exist exactly two cycles with $c_{k}^{2}\left(e_{i}^{*}\right)=1$, the collection of $c_{k}$ is a cycle 2-cover. By the proof of the previous Theorem 4.8 the corresponding ambient surface is $S^{2}$.

(2) Let $G \subset \mathbb{R}^{2}$ be an arbitrary graph. Again, we make it connected, if necessary, by adding bridges. Let $G^{\prime} \subset S^{2}$ be the preimage under the $2: 1$ cover $S^{2} \rightarrow \mathbb{R P}^{2}$.

If $G^{\prime}$ is disconnected then it has two components both isomorphic to $G$. Then $G$ is planar, (1) applies, and an embedding $G \hookrightarrow S^{2}$ defines a $\mathbb{Z}$-emm of type $A_{g}$. Consider the $g+1$ regions $S^{2} \backslash G$ and the corresponding cycles $c_{k}$. Since $g+1 \geq 5$ and the complete graph $K_{5}$ is non-planar, there exist two cycles $c_{k_{1}}, c_{k_{2}}$ which do not share an edge. Then the quadratic form $\frac{1}{2} \sum_{k \neq k_{1}, k_{2}} c_{k}^{2}+\frac{1}{2}\left(c_{k_{1}}-c_{k_{2}}\right)^{2}$ is a $\mathbb{Z}$-emm of $G$, and it is easy to check that it has type $D_{g}$.

Otherwise, $G^{\prime}$ is a connected graph of genus $2 g-1$. As above, $H^{1}\left(G^{\prime}, \mathbb{Z}\right)$ together with the quadratic form $\sum_{k=1}^{2 g} c_{k}^{2}$ is a root system of type $A_{2 g-1}$ in its realization as the hyperplane $\left\{\sum n_{k}=0\right\}$ in $\mathbb{Z}^{2 g}$.

On the homology, the antipodal involution $\iota$ can be written as $c_{k} \mapsto-c_{k+g}$, where we set $c_{m}:=c_{m-2 g}$ if $m>2 g$. The image of $H_{1}\left(G^{\prime}, \mathbb{Z}\right)$ in $H_{1}(G, \mathbb{Z})$ is the projection of $H_{1}\left(G^{\prime}, \mathbb{Z}\right)$ onto the $(+1)$-eigenspace $H_{1}^{+}\left(G^{\prime}, \mathbb{R}\right)$, and can be identified with the standard Euclidean $\mathbb{Z}^{g}$ with the basis $c_{1}, \ldots, c_{g}$. The homology group $H_{1}(G, \mathbb{Z})$ is the $\mathbb{Z}_{2}$-extension of it obtained by adding vector $\frac{1}{2}(1, \ldots, 1)$.

Thus, the dual lattice $\left(H^{1}(G, \mathbb{Z}), 2 q\right)$ can be identified with the sublattice of $\mathbb{Z}^{g}$ of integral vectors with even sum of coordinates. This is the standard definition of the $D_{g}$ lattice.

For the opposite direction, note that the quadratic form for $D_{g}$ is a sum of $g$ squares:

$$
\begin{aligned}
2 q & =2 \sum_{i=1}^{g} x_{i}^{2}-2 x_{1} x_{3}-2 x_{2} x_{3}-2 \sum_{i=3}^{g-1} x_{i} x_{i+1}= \\
& =\left(x_{1}+x_{2}-x_{3}\right)^{2}+\left(x_{1}-x_{2}\right)^{2}+\left(x_{3}-x_{4}\right)^{2}+\ldots\left(x_{g-1}-x_{g}\right)^{2}+x_{g}^{2}
\end{aligned}
$$

This gives $g$ cycles $c_{k}$ and thus a positive double cover by $g$ or $g+1$ simple cycles $d_{\ell}$, which defines an embedding of $G$ into $S^{2}$ or $\mathbb{R P}^{2}$, if $G$ is 2-connected and loopless. Finally, if $G$ is planar then it is moreover projective planar.

\section{Existence of $\mathbb{R}-$ emms}

Recall that by Lemma 2.7 it sufficient to construct $\mathbb{R}$-emms for cubic graphs. We begin by characterizing coedges. The following simple lemma will be useful:

Lemma 5.1. If $G$ is a connected bridgeless graph with the property that every edge is contained in a two element cutset then $G$ is cyclic. 
Proof. Suppose, as the induction hypothesis, $G$ is a minimal counterexample. Take any edge $e$ and a cutset $\{e, f\}$ containing it. This exhibits $G$ as a "cycle" $\left[e, G_{a}, f, G_{b}\right]$ with $G_{a}$ and $G_{b}$ disjoint and joined only by $e$ and $f$. Enlarge this cycle to exhibit $G$ as a larger "cycle", or "necklace" consisting of a chain of "gems" $G_{1}, G_{2}, \ldots G_{r}$ connected cyclically by single edges. Further assume that this necklace is maximal, so that no $G_{i}$ contains a $G_{i}$-bridge. Then by the induction hypothesis, each $G_{i}$ must be cyclic. Since we assumed $G$ was not cyclic, one of them contains an edge. That edge is not in any 2-element $G$-cutset, contradicting the choice of $G$.

We can now give the promised characterization of coedges:

Definition 5.2. A cycle in a graph $\Gamma$ will be called a $(0,1)$-cycle if all directed edges appear in it with coefficients in $\{+1,0,-1\}$; that is, the cycle is a sum of simple cycles with disjoint edge supports.

Lemma 5.3. A nonzero cocycle $z \in H^{1}(\Gamma, \mathbb{Z})$ is a coedge $\Longleftrightarrow z(c) \in\{+1,0,-1\}$ for all $(0,1)$-cycles $c$.

Proof. Clearly a coedge satisfies this condition, so we need to prove the converse.

We can assume that $\Gamma$ is 2-edge connected, i.e. connected and bridgeless. In a bridgeless graph, all edges are divided into equivalence classes by $e \sim e^{\prime}$ iff $e^{*}= \pm e^{\prime *}$. By contracting all but one edge in each equivalence class, we can assume that $\Gamma$ is 3 -edge connected.

We will proceed by induction on the number of edges in $\Gamma$. Choose some edge $e_{1}$ of $\Gamma$. We form a new graph $\Gamma \backslash e_{1}$ by deleting $e_{1}$. We note that $(0,1)$-cycles in $\Gamma \backslash e_{1}$ are $(0,1)$-cycles in $\Gamma$, and we have the natural pullback map $f: H^{1}(\Gamma, \mathbb{R}) \rightarrow H^{1}\left(\Gamma \backslash e_{1}, \mathbb{R}\right)$, so $f(z)$ satisfies the conditions of the lemma and by induction is a coedge if it is nonzero. But $\operatorname{ker}(f)=e_{1}^{*}$, so either $z=n e_{1}^{*}$ (in which case we immediately have $z= \pm e_{1}^{*}$, a coedge) or $z=n e_{1}^{*}+e_{2}^{*}$. In this case we claim that $n \in\{-1,0,1\}$. To show this we exhibit a simple cycle $c$ in $\Gamma \backslash e_{2}$ containing $e_{1}$. This is certainly possible as long as $e_{1}$ is not a bridge in $\Gamma \backslash e_{2}$. On the other hand, if $e_{1}$ is a bridge in $\Gamma \backslash e_{2}$ then $e_{1}^{*}= \pm e_{2}^{*}$ in $H^{1}(\Gamma, \mathbb{Z})$, so $f(z)=0$, and $z= \pm e_{1}^{*}$ as above. If $n=0$ we're done, so can assume that $z=e_{1}^{*}+e_{2}^{*}$ by changing the orientation of $e_{1}$ if necessary.

If $\Gamma \backslash\left\{e_{1}, e_{2}\right\}$ has a bridge $e_{3}$ then we get a 3 -term relation on coedges $e_{3}^{*}=e_{1}^{*} \pm e_{2}^{*}$ in $\Gamma$, implying either $z=e_{3}^{*}$, in which case we're done, or $z=2 e_{2}^{*}-e_{3}^{*}$, which would contradict $z(c) \in\{+1,0,-1\}$ for simple cycles $c$, by an argument similar to that given above. The alternative is that $\Gamma \backslash\left\{e_{1}, e_{2}\right\}$ is bridgeless, which we show is impossible.

Assume $\Gamma \backslash\left\{e_{1}, e_{2}\right\}$ is bridgeless. Delete any edge $e \neq e_{1}, e_{2}$. Induction tells us that $z$ becomes a coedge $e_{3}^{*}$ in $\Gamma \backslash e$, so we have a four term relation $e_{1}^{*}+e_{2}^{*}=e_{3}^{*}+k e$ in $\Gamma$. Thus we have a four element cutset $\left\{e_{1}, e_{2}, e, e_{3}\right\}$ on $\Gamma$, and so a two element cutset $\left\{e, e_{3}\right\}$ on $\Gamma \backslash\left\{e_{1}, e_{2}\right\}$ for any $e$. But by the previous lemma 5.1, the only graphs where every edge is contained in a two element cutset are cyclic graphs, and if $\Gamma \backslash\left\{e_{1}, e_{2}\right\}$ was a cyclic graph we could easily find a $(0,1)$-cycle $c$ in $\Gamma$ such that $\left(e_{1}^{*}+e_{2}^{*}\right)(c)=2$, a contradiction.

Theorem 5.4. Any bridgeless cubic graph $G$ admits a strong $\mathbb{Q}$-emm.

Proof. We will reduce the problem to the existence of strong $\mathbb{Q}$-emms on certain strictly smaller graphs. In this way, we get an inductive construction of such forms. Let $e_{0}$ be an edge in $G$. We can produce 3 graphs on fewer vertices by modifying the region of $G$ containing $e_{0}$ as shown on Figure 2. Note that $G_{1}$ and $G_{2}$ are analogous to each other. We call the two edges formed by this process $e_{1}$ and $e_{2}$ in each of $G_{1}, G_{2}, G_{3}$, and we orient them as shown. 

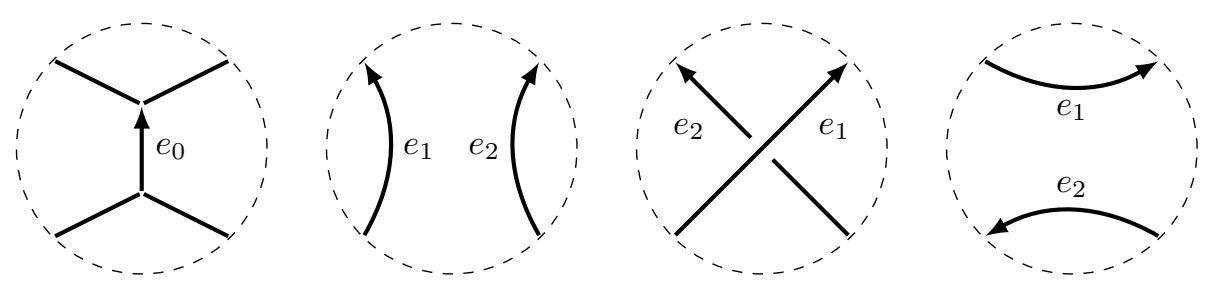

Figure 2. The graphs $G, G_{1}, G_{2}, G_{3}$

Since integral cycles in $G_{i}$ lift to integral cycles in $G$, we have maps $H_{1}\left(G_{i}, \mathbb{Z}\right) \rightarrow$ $H_{1}(G, \mathbb{Z}), i \in\{1,2,3\}$. Hence we get the opposite maps $\phi_{i}: H^{1}(G, \mathbb{Z}) \rightarrow H^{1}\left(G_{i}, \mathbb{Z}\right)$. We note the following:

Claim 5.5. For a cocycle $z \in H^{1}(G, \mathbb{Z})$, if $\phi_{i}(z)$ is a coedge for each $i \in\{1,2,3\}$ then $z$ is itself a coedge.

Proof. This follows immediately from the Lemma 5.3 and the observation that every $(0,1)$ cycle in $G$ corresponds to a $(0,1)$-cycle in at least one of the $G_{i}$

Examining these maps more closely, we see that the kernels of $\phi_{i}$ are generated by the cocycles shown in Figure 3. We also have maps on quadratic forms $\psi_{i}: \operatorname{Sym}^{2} H_{1}\left(G_{i}, \mathbb{Z}\right) \rightarrow$ $\operatorname{Sym}^{2} H_{1}(G, \mathbb{Z})$. By the induction hypothesis we have strong $\mathbb{Q}$-emms $q_{i}$ on $G_{i}$. These lift to forms $\psi_{i}\left(q_{i}\right)$ on $G$, positive semidefinite and zero only on $\operatorname{ker}\left(\phi_{i}\right)$. We wish to build a strong emm as a convex combination $x_{1} \psi_{1}\left(q_{1}\right)+x_{2} \psi_{2}\left(q_{2}\right)+x_{3} \psi_{3}\left(q_{3}\right)$ where $x_{1}+x_{2}+x_{3}=1, x_{i} \geq 0$.
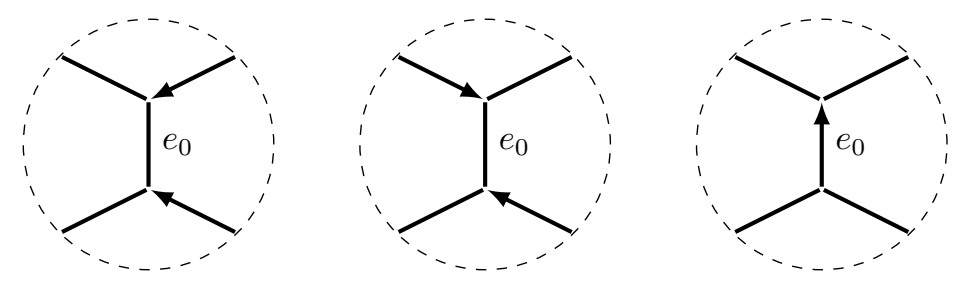

FiguRE 3. Generators of $\operatorname{ker} \phi_{1}, \operatorname{ker} \phi_{2}, \operatorname{ker} \phi_{3}$

Assume first that $G_{i}$ are bridgeless (the special cases where some of the $G_{i}$ are not bridgeless will be dealt with later). Note that for every edge $e \neq e_{0}$ we have $\psi_{i}\left(q_{i}\right)\left(e^{*}\right)=1$ because $\phi_{i}$ maps coedges to coedges. For every other (that is noncoedge) integral cocycle $z$ not in $\operatorname{ker}\left(\phi_{i}\right)$ we have $\psi_{i}\left(q_{i}\right)(z) \geq 1$, with at least one of the $\psi_{i}\left(q_{i}\right)(z)>1$ by Claim 5.5.

Hence if $x_{i} \neq 0$ for all $i$, we need only to verify the emm conditions on the three cocycles generating $\operatorname{ker}\left(\phi_{i}\right)$. For brevity we write $c_{i}=q_{i}\left(e_{1}^{*}+e_{2}^{*}\right)$ and note that since $q_{i}$ is a quadratic form with $q_{i}\left(e_{1}^{*}\right)=q_{i}\left(e_{2}^{*}\right)=1$ we must have $q_{i}\left(e_{1}^{*}-e_{2}^{*}\right)=4-c_{i}$. Hence, if $e_{1}^{*} \pm e_{2}^{*} \neq 0$ in $G_{i}$ then $c_{i} \in[1,3]$, with $c_{i} \in(1,3)$ if $e_{1}^{*} \pm e_{2}^{*}$ are not coedges. We can now write the (strong) emm conditions as follows:

(1) $x_{2}\left(4-c_{2}\right)+x_{3}\left(4-c_{3}\right) \geq 1$ when $\operatorname{ker}\left(\phi_{1}\right) \neq\{0\}$ and with equality only when the generator of $\operatorname{ker}\left(\phi_{1}\right)$ is a coedge.

(2) $x_{1}\left(4-c_{1}\right)+x_{3} c_{3} \geq 1$ when $\operatorname{ker}\left(\phi_{2}\right) \neq\{0\}$ and with equality only when the generator of $\operatorname{ker}\left(\phi_{2}\right)$ is a coedge.

(3) $x_{1} c_{1}+x_{2} c_{2}=1\left(\operatorname{ker}\left(\phi_{3}\right)\right.$ is generated by a coedge, namely $e_{0}^{*}$.) 
Note that these inequalities are symmetric except for the symbols $\geq,=$. Consider the generic case where $c_{1}, c_{2}, c_{3} \in(1,3)$ (we'll deal with the nongeneric cases later). We use three solution types, depending on the values of $c_{1}, c_{2}, c_{3}$ :

Case 1. When $\left(c_{2} \leq 2\right.$ or $\left.c_{3} \leq 2\right)$ and $\left(c_{1} \leq 2\right.$ or $\left.c_{3} \geq 2\right)$ set $x_{1}=x_{2}=\frac{1}{c_{1}+c_{2}}$. Consider the left hand side of the first inequality, call it $k$. We have $k=\frac{4-c_{2}}{c_{1}+c_{2}}+\frac{\left(c_{1}+c_{2}-2\right)\left(4-c_{3}\right)}{c_{1}+c_{2}}$. Since $c_{3}<3$ we have $k>\frac{2+c_{1}}{c_{1}+c_{2}} \geq 1$ when $c_{2} \leq 2$. If $c_{3} \leq 2$ then $k \geq \frac{2 c_{1}+c_{2}}{c_{1}+c_{2}}>1$. The other half of the conjunction follows by symmetry.

Case 2. When $c_{1} \geq 2$ and $c_{3}<2$, set $x_{2}=\epsilon c_{1}, x_{1}=1 / c_{1}-\epsilon c_{2}$ for small $\epsilon>0$. Indeed, if we take $\epsilon=0$ the left hand side of the first inequality simply becomes $\frac{\left(c_{1}-1\right)\left(4-c_{3}\right)}{c_{1}}$, which is greater than 1 . The second inequality is also satisfied (see case 1). Of course this doesn't quite work, since $x_{2}=0$, but by continuity the conditions still hold for suitably small $\epsilon$.

Case 3. When $c_{2} \geq 2$ and $c_{3}>2$, set $x_{1}=\epsilon c_{2}, x_{2}=1 / c_{2}-\epsilon c_{1}$. This works by symmetry with case 2 .

Hence all the generic cases are solved. Consider now the exceptional cases. That is, assume that either at least one of the $G_{i}$ has a bridge, or that one of the $c_{i}$ is $0,1,3$, or 4. By listing all possible such cases, we will show that they are of only three types (up to symmetry), shown in Figure 4.
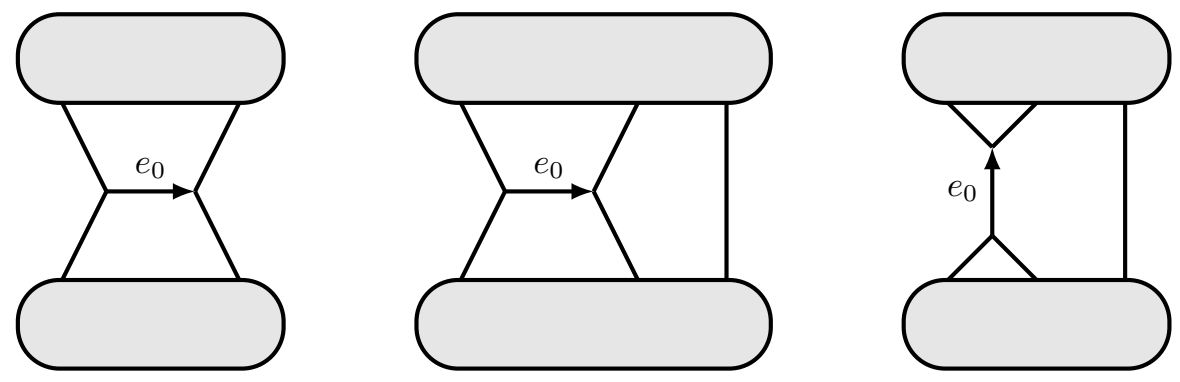

Figure 4. Exceptional cases A, B, C

(1) Some $c_{i} \in\{0,4\}$

(a) $c_{1}=0$ or $c_{2}=0$. This cannot happen in a bridgeless graph.

(b) $c_{3}=4$. This occurs only in graphs of type A.

(c) $c_{3}=0$. This is symmetric to $c_{3}=4$

(d) $c_{1}=4$. This happens exactly when $c_{3}=0$.

(e) $c_{2}=4$. This happens exactly when $c_{3}=4$.

(2) Some $G_{i}$ contains a bridge.

(a) $G_{1}$ contains a bridge. This is the situation of type B.

(b) $G_{2}$ contains a bridge. Symmetric to $G_{1}$ containing a bridge.

(c) $G_{3}$ contains a bridge. This is type C.

(3) Some $c_{i} \in\{1,3\}$. That is, one of $e_{1}^{*} \pm e_{2}^{*}$ is a coedge.

(a) $c_{2}=1$. This is type $\mathrm{C}$. 
(b) $c_{2}=3$. This is type B.

(c) $c_{1} \in\{1,3\}$. This is symmetric to $c_{2} \in\{1,3\}$.

(d) $c_{3}=3$. This is type B.

(e) $c_{3}=1$. This is symmetric to $c_{3}=3$.

If we choose $e_{0}$ to not lie in any 2-element cutset, then it becomes unnecessary to deal with case C. This is always possible, since by Lemma 5.1 every edge is part of a two element cutset only if $G$ is cyclic. We proceed to construct strong emms for the remaining two cases:

(A) We observe that $\operatorname{ker}\left(\phi_{1}\right)=\{0\}$. Moreover, since every $(0,1)$ cycle in $G$ is of the form $\phi_{1}(c)$ for some $(0,1)$ cycle $c$ in $G_{1}$, by the Lemma 5.3 every noncoedge in $H^{1}(G, \mathbb{Z})$ ) maps through $\phi_{1}$ to a noncoedge in $H^{1}\left(G_{1}, \mathbb{Z}\right)$. Hence $\psi_{1}\left(q_{1}\right)$ satisfies the conditions for a strong emm.

(B) We have $c_{2}=c_{3}=3$; we construct the form $\frac{1}{3} \psi_{2}\left(q_{2}\right)+\frac{2}{3} \psi_{3}\left(q_{3}\right)$. We need to show that this is greater than 1 on all integral noncoedges. Equivalently (by Lemma 5.3), we need to show that for any $z \in H^{1}(G, \mathbb{Z})$ with $z(c)>1$ for some $(0,1)$-cycle $c$ there is an $i \in\{2,3\}$ and $(0,1)$-cycle $c^{\prime}$ in $G_{i}$ such that $\phi_{i}(z)\left(c^{\prime}\right)>1$.

We may assume that $c$ is the sum of at most two simple cycles (if $z\left(\sum c_{i}\right)>1$ then $z\left(c_{1}\right)>1$ or $z\left(c_{1}+c_{2}\right)>1$ for some $\left.c_{1}, c_{2}\right)$. Since $c$ is a $(0,1)$ cycle it must be the image of a cycle in at least one of $G_{1}, G_{2}, G_{3}$. If it is the image of a cycle in $G_{2}$ or $G_{3}$ there is nothing to show, so we can assume that it is the image of some cycle $k$ in $G_{1}$, but not of any cycle in $G_{2}$ or $G_{3}$. In order for this to be true $c$ must contain the edge $e_{0}$ and not the edge that becomes a bridge in $G_{1}$.

By symmetry assume that $k$ contains the edge $e_{1}$ and not $e_{2}$. Note that since $c$ was a sum of 2 (possibly trivial) simple cycles, so is $k$, say $k=k_{1}+k_{2}$ where $k_{1}$ the simple summand containing $e_{1}$. Note that, since $k_{2}$ contains neither $e_{1}$ nor $e_{2}$, the image of $k_{2}$ in $G$ is also the image of simple cycles in $G_{2}$ and $G_{3}$, so if $\phi(z)\left(k_{2}\right)>1$ the problem would be solved immediately. Hence we will further assume $\phi(z)\left(k_{1}\right) \neq 0$.

We will proceed to use $k$ to construct a $(0,1)$ cycle $k^{\prime}$ in $G_{1}$ that contains both $e_{1}$ and $e_{2}$ and has $\phi_{1}(z)\left(k^{\prime}\right)>1$. If we can succeed in doing this the result will follow, because depending on the relative orientations of $e_{1}$ and $e_{2}$ in $k^{\prime}$ there must be either a $(0,1)$ cycle $c^{\prime}$ in $G_{2}$ that maps to the same cycle in $G$ as $k^{\prime}$ does (so $\phi_{2}(z)\left(c^{\prime}\right)=\phi_{1}(z)\left(k^{\prime}\right)>1$ ) or one in $G_{3}$ that maps to the same cycle as $k^{\prime}$ (so $\phi_{3}(z)\left(c^{\prime}\right)>1$ ).

To build $k^{\prime}$, find a simple cycle $l$ passing through $e_{2}$ that intersects $k_{2}$ in a (possibly empty) arc (continuous path of edges). Such a cycle always exists, since given any cycle containing $e_{2}$ (these exist by connectedness) there is a maximal arc (possibly the whole cycle) disjoint from $k_{2}$, and a (possibly empty) arc in $k_{2}$ joining its endpoints. We can choose the arc in $k_{2}$ such that $l+k_{2}$ is also a $(0,1)$ cycle. But now since $\phi_{1}(z)(k)>1$, out of the four $(0,1)$ cycles $k_{1} \pm l, k_{1} \pm\left(l+k_{2}\right)$ one of $\phi_{1}(z)\left(k_{1} \pm l\right), \phi_{1}(z)\left(k_{1} \pm\left(l+k_{2}\right)\right)$ must be greater than 1. (Indeed, recalling our assumption that $\phi_{1}(z)\left(k_{1}\right) \neq 0$, if $\phi_{1}(z)(l) \neq 0$ one of $k_{1} \pm l$ will work, if $\phi_{1}(z)\left(k_{2}\right) \neq 0$ one of $k_{1} \pm\left(l+k_{2}\right)$ will work, otherwise all four work .) Calling this cycle $k^{\prime}$ the result follows.

Hence the nongeneric cases are resolved. This concludes the proof of Theorem 5.4.

Remark 5.6. In the induction argument of Theorem 5.4, it may happen that some of the incoming and outgoing edges are in fact the same. Then one of the graphs $G_{i}$ may have 
a component which is a "loop with one edge $e$ and zero vertices". We deal with this case formally, by taking $e^{2}$ to be the corresponding quadratic form.

Theorem 5.7. Any graph $G$ admits a strong $\mathbb{Q}$-emm.

Proof. Existence of an $\mathbb{Q}$-emm follows at once from Theorem 5.4 and Lemma 2.7 which reduces graph $G$ to a disjoint collection $G^{\prime}=\sqcup G_{k}^{\prime}$ of cubic graphs. However, the inclusion $S(G) \subset S\left(G^{\prime}\right)$ (see Definition 3.1) may be strict, so a strong $\mathbb{Q}$-emm $q$ of $G^{\prime}$ may not be a strong $\mathbb{Q}$-emm of $G$.

By the result [ER94, 4.1] which we mentioned in Remark 3.6, the distinct vectors $e_{i}^{* 2}$ for the graph $G^{\prime}$ are linearly independent. Thus, there exists a $q_{0} \in M_{\mathbb{Q}}$ such that $q_{0}$ is zero on $S(G)$ and positive on $S\left(G^{\prime}\right) \backslash S(G)$. Then $q+\epsilon q_{0}$ for $0<\epsilon \ll 1$ is a required strong $\mathbb{Q}$-emm for $G$.

\section{Concluding remarks and generalizations}

6.1. Characterization of $\mathbb{Z}$-emms of type $E_{n}$. It would be interesting to find a geometric characterization of graphs admitting $\mathbb{Z}$-emms of types $E_{6}, E_{7}, E_{8}$, similar to the characterization for $A_{n}$ and $D_{n}$ given in Theorem 4.2.

6.2. Special quadratic forms, and physical interpretation. For any collection of positive real numbers $\left(\lambda_{1}, \ldots, \lambda_{m}\right)$ there is a natural positive definite quadratic form $Q=$ $\sum \lambda_{i} e_{i}^{* 2}$ on the homology group $H_{1}(G, \mathbb{R})$. Since $Q$ is nondegenerate, we can use it to identify $H_{1}$ and $H^{1}$, thus producing a positive definite quadratic form $q$ on $H^{1}$. In coordinates, the matrix of $q$ is the inverse of the matrix of $Q$. Searching for an $\mathbb{R}$-emm of this form leads to a system of $m$ nonlinear equations in $m$ variables which seems to be hard to solve. We note that our solution for an $\mathbb{R}$-emm is not of this special form.

One can make a graph into an electric network by putting resistors $\lambda_{i}$ along the edges $e_{i}$. The total energy dissipation of this electric system is $Q$. The condition $q\left(e_{i}^{*}\right)=1$ can be reformulated in these terms as follows. For any edge $e_{i}$, let $G_{i}$ be the graph obtained by cutting the edge $e_{i}$ in the middle, thus producing two end points $p_{i}, q_{i}$. Then the condition is that the resistance of $G_{i}$ between the points $p_{i}$ and $q_{i}$ is 1 , for each $i=1, \ldots, m$.

6.3. All dicing 2nd Voronoi cones. The method of the proof of Theorem 5.4 may also apply to arbitrary, not necessarily cographic, regular matroids. This would give a bigger open set in which $\overline{\mathrm{A}}_{g}^{\text {perf }}$ and $\overline{\mathrm{A}}_{g}^{\mathrm{vor}}$ coincide.

6.4. Other Torelli maps. The proofs of Theorems 3.4, 3.7, 3.8 work in a more general situation, if we replace $S(G)$ by any finite set $\left\{v^{* 2}\right\}$ of symmetric rank 1 tensors. Thus, they give regularity criteria for any rational map $\overline{\mathrm{M}} \rightarrow \overline{\mathrm{A}}_{g}^{\tau}, \tau=\tau^{\mathrm{vor}}, \tau^{\text {perf }}, \tau^{\text {cent }}$, for as long as $\overline{\mathrm{M}}$ is toroidal and the monodromy map has a specific form $r_{i} \mapsto v_{i}^{* 2}$. For example, once properly set up, this may apply to intermediate jacobians of cubic 3 -folds.

\section{References}

$\left[\right.$ ALT $\left.^{+} 10\right]$ Valery Alexeev, Ryan Livingston, Joe Tennini, et al., Extended Torelli map to the Igusa blowup in genus 6, 7, and 8, arXiv: math/1105.4384.

[Ale02] Valery Alexeev, Complete moduli in the presence of semiabelian group action, Ann. of Math. (2) 155 (2002), no. 3, 611-708. 
[Ale04] Compactified Jacobians and Torelli map, Publ. Res. Inst. Math. Sci. 40 (2004), no. 4, $1241-1265$.

[AMRT75] A. Ash, D. Mumford, M. Rapoport, and Y. Tai, Smooth compactifications of locally symmetric varieties, Lie groups: history, frontiers and applications, vol. IV, Math Sci Press, 1975.

[Arc80] Dan Steven Archdeacon, A Kuratowski theorem for the projective plane, ProQuest LLC, Ann Arbor, MI, 1980, Thesis (Ph.D.)-The Ohio State University.

[Arc81] _ A Kuratowski theorem for the projective plane, J. Graph Theory 5 (1981), no. 3, 243246.

[BC76] E. S. Barnes and M. J. Cohn, On the inner product of positive quadratic forms, J. London Math. Soc. (2) $12(1975 / 76)$, no. 1, 32-36.

[ER94] R. M. Erdahl and S. S. Ryshkov, On lattice dicing, European J. Combin. 15 (1994), no. 5, 459-481.

[ER01] Robert Erdahl and Konstantin Rybnikov, Voronoi-Dickson hypothesis on perfect forms and L-types, arXiv: math/0112097 (2001), 26pp.

[ER02] On Voronoi's two tilings of the cone of metrical forms, Rend. Circ. Mat. Palermo (2) Suppl. (2002), no. 70, part I, 279-296, IV International Conference in "Stochastic Geometry, Convex Bodies, Empirical Measures \& Applications to Engineering Science", Vol. I (Tropea, 2001).

[FC90] G. Faltings and C.-L. Chai, Degenerations of Abelian Varieties, vol. 22, Ergebnisse der Mathematik und ihrer Grenzgebiete, no. 3, Springer-Verlag, 1990.

[Igu67] Jun-ichi Igusa, A desingularization problem in the theory of Siegel modular functions, Math. Ann. 168 (1967), 228-260.

[Nam73] Yukihiko Namikawa, On the canonical holomorphic map from the moduli space of stable curves to the Igusa monoidal transform, Nagoya Math. J. 52 (1973), 197-259.

[Nam76] _ A new compactification of the Siegel space and degeneration of Abelian varieties. I,II, Math. Ann. 221 (1976), no. 2,3, 97-141, 201-241.

[Ox192] James G. Oxley, Matroid theory, Oxford Science Publications, The Clarendon Press Oxford University Press, New York, 1992.

[SB06] N. I. Shepherd-Barron, Perfect forms and the moduli space of abelian varieties, Invent. Math. 163 (2006), no. 1, 25-45.

[Vor09] G. Voronoi, Nouvelles applications des paramètres continus à la théorie des formes quadratique, I,II,III, J. Reine Angew. Math. 133, 134, 136 (1908-09), 97-178, 198-287, 67-181.

Department of Mathematics, University of Georgia

E-mail address: valery@math.uga.edu, brunyate@math.uga.edu 